\title{
Propuesta para mejorar el proceso de impresión en la industria litográfica
}

\section{A Proposal for improvement of print process in lithographic industry}

DOI: http://dx.doi.org/10.17988/bilo

Artículo de Investigación Científica. Fecha de Recepción: 30/05/2020. Fecha de Aceptación: 13/06/2020

\author{
Lorena Henríquez-Sarmiento \\ Universidad de la costa CUC, Barranquilla, (Colombia) \\ lenriqu8@cuc.edu.co \\ Sebastián Camacho-Menendez \\ Universidad de la costa CUC, Barranquilla, (Colombia) \\ scamacho@cuc.edu.co \\ Alexander Troncoso-Palacio \\ Universidad de la costa CUC, Barranquilla, (Colombia) \\ atroncos1@cuc.edu.co
}

Para citar este artículo:

A. Lombana-Jiménez, L. Sarmiento-Escobar y A. Troncoso-Palacio, "Medición del servicio de internet durante un mes de clases presenciales asistidas a través de tecnología remota”, INGE CUC, vol. 2, no. 1, 2020. DOI: http://dx.doi.org/10.17987/bilo

\section{Resumen}

En el presente artículo, se utilizaron algunas herramientas de calidad para identificar problemas presentados en el sector litográfico. Con las cuales se descubrió que los retrasos en los pedidos de producción se debieron a problemas de impresión, donde se encontraron tres fallas comunes. Primera. El 34\% de la producción con Impresión corrida. Segundo. El 23\% con Papel mal cortado. Tercero. El 3\% Impresión incompleta. Por lo tanto, se propusieron las siguientes recomendaciones. Realizar periódicamente mantenimientos para reducir el daño causado a la impresión y evitar la obstrucción de los cabezales de las máquinas de impresión. Además, se debería asignar a una persona como responsable de inspeccionar el proceso para evitar que se envíen folletos incompletos a los clientes. Al finalizar este proyecto, se concluyó que, al aplicar algunas herramientas de calidad, estas facilitan enormemente el trabajo, encontrando las causas de los problemas y las no conformidades presentadas durante el proceso de impresión, lo que ayuda a la mejora continua y poder lograr la satisfacción de los clientes.

Palabras clave: Calidad Litográfica; Diagrama de Ishikawa; Herramientas de Control; Problemáticas litográficas.

\begin{abstract}
This paper, quality tools some were used to identify problems presented in the lithographic sector. with them it was found presented delays in the production orders were due to printing problems, where three common flaws were found. First. Ink stained print. Second. Poorly cut paper. Third. Incomplete print. Therefore, the next recommendations were proposed. Maintenance should be carried out periodically to mitigate the damage caused to the printing and to avoid clogging of the heads of the printing machines. In addition, a person should be assigned as responsible for inspecting the process to prevent incomplete brochures from being sent to customers. When finished this project concluded that, applying some
\end{abstract}


quality tools, these greatly facilitate the work, finding the causes of the problems and nonconformities presented during the printing process, which helps continuous improvement and being able to achieve the satisfaction of the customers.

Key Words: Control tools; Ishikawa's diagram; Lithographic problems; Lithographic quality

\section{INTRODUCCIÓN}

La aplicación de las herramientas de calidad dentro de los procesos es de gran importancia debido a que a través de ellas se puede identificar diferentes problemáticas e inconformidades en los procesos como en [1, 2, 3], encontraron las causas de los defectos en procesos de extrusión de plásticos. Con la intención de mantenerse durante el tiempo en los mercados, muchas compañías invierten cantidades de dinero en la implementación de herramientas de control de la calidad en sus procesos, con el fin de lograr la satisfacción de los clientes, a pesar de las innumerables ventajas que puede traer el control de la calidad esta es muy costosa por la cantidad de recursos necesarios para que su implementación, sea completamente exitosa. Por esto, se hace una breve revisión sobre este tema.

\section{BREVE REVISIÓN DE LA LITERATURA}

Algunas investigaciones como [4], hacen referencia a la aplicación de las herramientas de calidad para el análisis y el control de problemas e inconformidades, con el fin de poder detectarlos a tiempo para poder tomar decisiones de manera oportuna y así obtener un aseguramiento en la calidad de su servicio. También en [5], Se aplicó un gráfico para identificar los productos que presentaban mayor inconformidad y el diagrama de Ishikawa se realizó para clasificar las distintas causas potenciales que hacen que las cartillas impresas sean inutilizable es decir pérdida de recursos y materia prima, través de estas herramientas de calidad se pudo observar que cual era la causa principal de los problemas más comunes presentados en la empresa, y también por medio de la encuesta realizada al dueño de esta empresa, se detectó que muchos de los clientes quedaban inconforme con resultado final. Con el Diagrama de Ishikawa se estudió el proceso de impresión de cuadernillos PRE-ICFES, en el cual se colocaron las causas y efectos de productos no conformes o cuadernillos inutilizables, según [6] El diagrama de Ishikawa es el resultado de la aplicación del método de análisis causa-efecto. Es una forma de encontrar, organizar y representar las diferentes causas que generan un problema. Los diagramas de Ishikawa reflejan este método con la finalidad de encontrar las causas de las fallas y los efectos que las mismas producen, de una forma sencilla. Por lo que, para hablar del desarrollo o introducción de un sistema de diagnóstico integral, se hace necesario la utilización de esta técnica. Según [7] "El gráfico de torta es la visualización fundamental. método que se empleó con frecuencia en los informes análisis de mapeo de datos categóricos en un visual mostrar en forma simple x e y para numérico comparaciones Opera en un conjunto de datos altamente agregado y solo es capaz de interpretar lo simple información. En informes comerciales, el analista de datos generalmente utiliza el esquema de codificación de color y el etiquetado de datos en la torta gráfico para descubrir dimensiones adicionales de datos comerciales y mejorar la legibilidad del gráfico para los usuarios, Sin embargo, el gráfico de torta en su naturaleza es muy básico (unidimensional) visualización que simplemente cumple con lo básico requisito de crecimiento exponencial y complejo dimensiones de los datos comerciales contemporáneos". [8,9,10]. El Grafico de torta es una herramienta que se utilizó para resumir los datos según su categoría y ver los productos que presentan más inconformidades y de qué tipo son, ya sea mal corte, tinta corrida o cuadernillos incompletos y así poder sugerirle a la empresa estrategias para disminuir la pérdida de materia prima. Mediante la encuesta realizada al dueño de la empresa, la cual es una herramienta investigativa que ayuda a mirar cuales son las fallas o si existe algún problema, o en su defecto ayuda a ver qué tan factible es o no realizar algo, en el caso de este artículo se le hicieron preguntas al dueño sobre los clientes, los pedidos y parte productiva y se detectó que existen ordenes atrasadas y aun no encuentran la causa de esta problemática por lo cual a través de la implementación de todas estas herramientas se pudo brindarle sugerencias para dar solución a posibles causas de esta problemática. En este artículo no solo se quiere reducir el tiempo, los recursos, y las no conformidades presentadas en la empresa a través de la utilización de las herramientas de calidad para la mejora continua de sus procesos, sino que también se desea obtener una mejora en la eficiencia y eficacia de esta para obtener así una mayor productividad logrando siempre la satisfacción de sus clientes.

\section{METODOLOGIA}

Para esta investigación, se utilizó una metodología cuantitativa, desarrollada como caso de estudio en una compañía que imprime cartillas, donde se decidió estudiar las causas por las cuales se presentan inconformidades, por lo cual se realizó una investigación de la parte productiva de la empresa y el por qué ocurren este tipo de problemas en la empresa, para lo cual se recopilara información a través de una encuesta, luego se categorizaran los productos no conforme de acuerdo a la falla presentada, y por último se realizara un diagrama de Ishikawa en donde se observaran causa y efectos de la problemática presentada para finalmente dar las respectivas sugerencias según las posibles soluciones que se puedan determinar para mejorar los procesos en esta empresa, como se ilustra en la figura 1. 


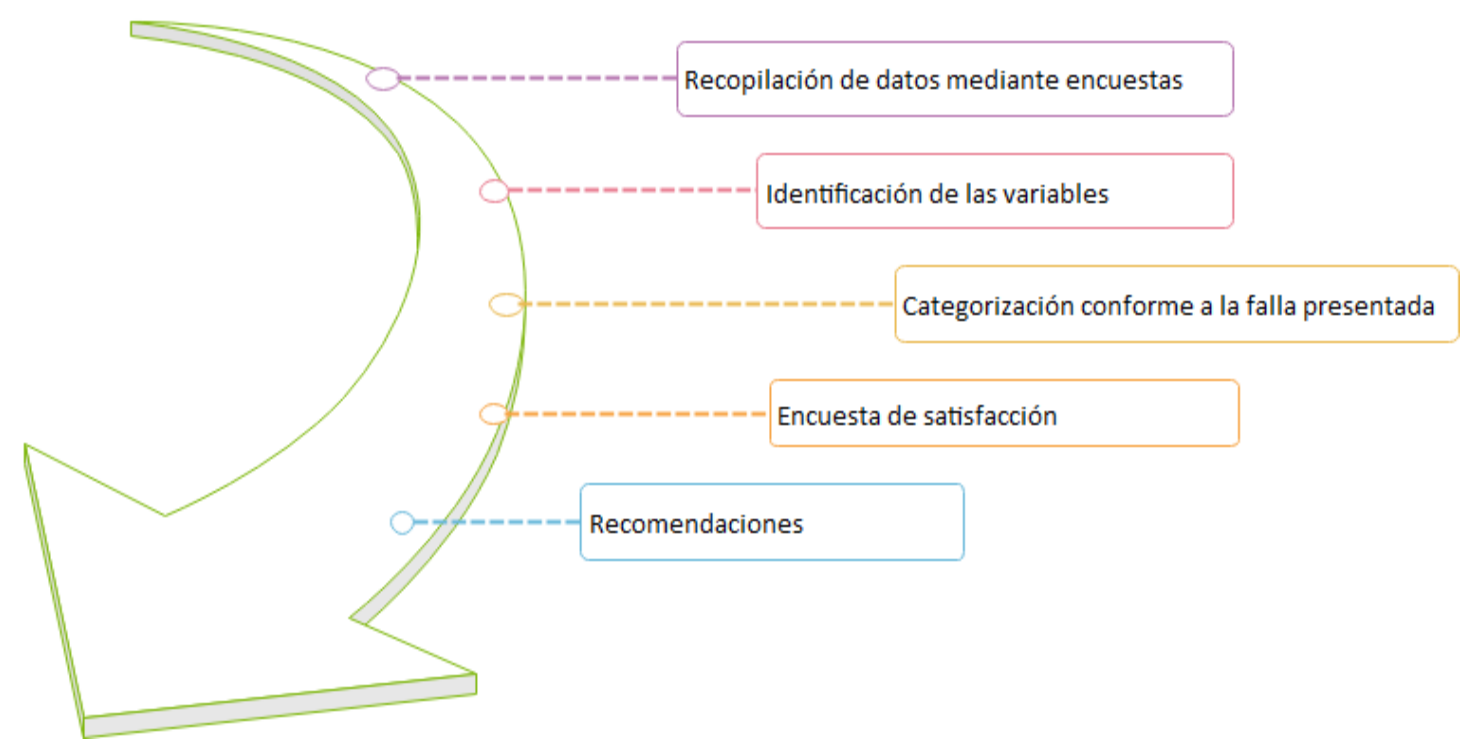

Figura 1: Metodología representada gráficamente.

\section{DESARROLLO}

Esta sección, Se realizó la recopilación de información de las cartillas mediante la categorización de esta mediante el grafico de torta de tal manera que se observe cuáles son las cartillas que presentan mayor informidad y de qué tipo son: Cabe resaltar que para efectos del presente artículo se escogió una muestra representativa de la producción de un lote de cartillas. En la figura 2 se observa la identificación de los defectos.

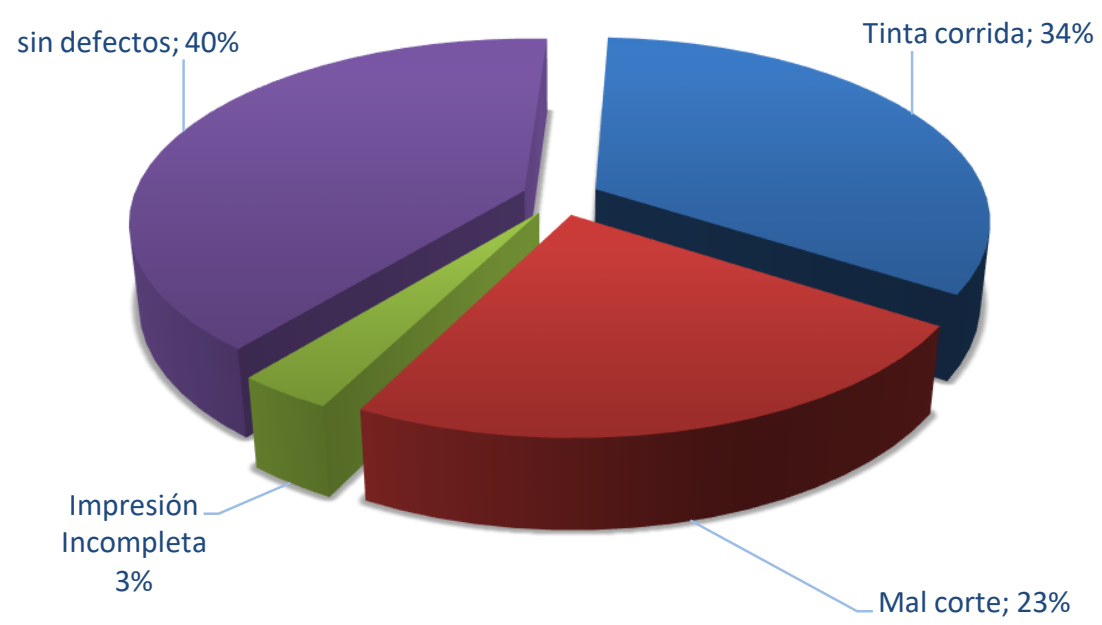

Gráfico 2. Identificación de defectos.

Con base en los resultados obtenidos, se procede a la realización de un diagrama causa efecto 


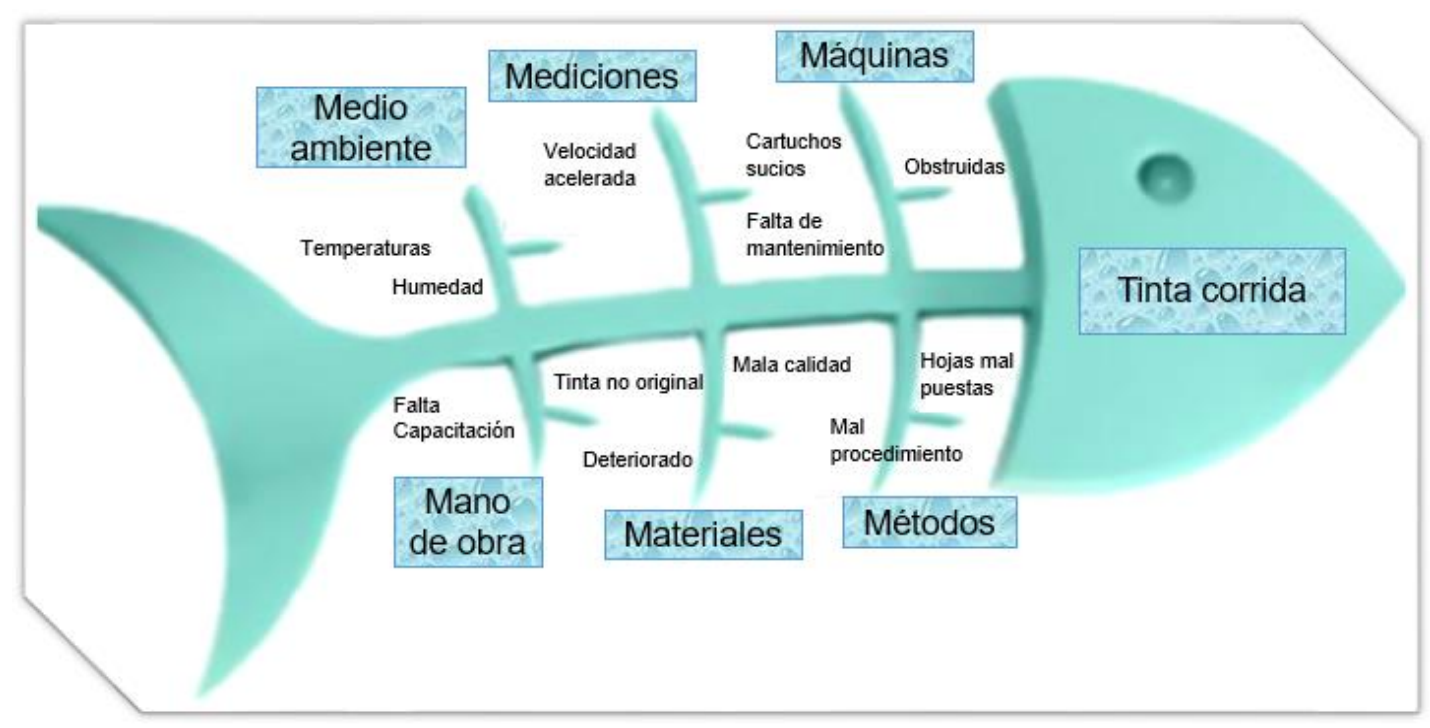

Grafico 3 Causas que ocasionan el defecto de tinta corrida

Luego se procede a la tabulación de las encuestas realizadas antes y después de la propuesta los resultados se observan en la tabla 1

\begin{tabular}{lcc}
\hline Marca temporal & Antes & Después \\
\hline $\begin{array}{l}\text { Los clientes se encuentran satisfechos con } \\
\text { la calidad de los productos }\end{array}$ & Satisfecho & muy satisfecho \\
$\begin{array}{l}\text { Hay pedidos no entregados a la fecha de } \\
\text { hoy }\end{array}$ & No & No \\
$\begin{array}{l}\text { ¿Alguna vez se han presentado demoras en } \\
\text { la entrega de pedidos? }\end{array}$ & Si & No \\
$\begin{array}{l}\text { Aproximadamente cuanto producto no } \\
\text { conforme queda después de un proceso de } \\
\text { elaboración de cartillas }\end{array}$ & 26 hojas salidas de \\
$\begin{array}{l}\text { Cuáles son las máquinas que son necesarias de } \\
\text { para un proceso de elaboración de cartillas } \\
\text { ¿Estas máquinas están en óptimas } \\
\text { condiciones? }\end{array}$ & Copiadoras \\
$\begin{array}{l}\text { Sí respondió no en la pregunta pasada } \\
\text { especifique cuales son estas maquinas } \\
\begin{array}{l}\text { ¿Los empleados están capacitados para } \\
\text { realizar todos los procesos? }\end{array}\end{array}$ & Si & Copiadoras \\
\hline
\end{tabular}

Tabla 1. Encuestas realizadas antes y después de aplicar los cambios

\section{RECOMENDACIONES Y CONCLUSIONES}

Luego del análisis realizado a través de las herramientas de calidad presentadas anteriormente como fue el grafico de torta y el diagrama de Ishikawa, se pudo llegar a las siguientes recomendaciones. También se deben realizar mantenimientos de manera periódica a fin de mitigar los daños causados en las cartillas y evitar la obstrucción de los cabezales. Mantener el papel en un lugar con buenas condiciones para evitar el deterioro y que estos se llenen de polvo. Contratar a un empleado que se encargue de revisar todas las cartillas, para evitar que así se envíen a los clientes cartillas incompletas. Capacitar de manera adecuada al operario encargado de los cortes para maneje de manera adecuada la máquina y así se disminuya el número de cartillas mal cortadas.

Las anteriores recomendaciones se le brindaron a la empresa con el fin de disminuir las fallas en los distintos procesos que se ven más afectados en la compañía. Es por esto que se puede concluir que el uso de las herramientas de control de la calidad son muy acertadas, ya que estas facilitan enormemente el trabajo que conlleva resolver los problemas e inconformidades presentados en una empresa, gracias al diagrama de torta y diagrama causa efecto o de Ishikawa se pudieron identificar satisfactoriamente todas la variables que afectaban la calidad del producto que oferta la empresa y 
posteriormente se llegó a una solución óptima y se llegó pronosticar que se podría reducir las inconformidades y aumentaría la satisfacción de los clientes y su confianza, donde a su vez se reducen tiempo y recursos utilizados.

\section{AGRADECIMIENTOS}

Esta investigación es el resultado de un proyecto de aula de la asignatura Control de la Calidad, periodo 2020-1 del programa de Ingeniería Industrial. Por lo cual, los autores desean agradecer principalmente a Dios por permitir la realización de este proyecto, a la Universidad de la Costa por haber facilitado las herramientas investigativas necesarias a lo largo de este proceso, a los revisores de este documento y por ultimo al Boletín Científico en Innovación Logística y Operaciones por la publicación. Muchas gracias a todos.

\section{Referencias}

[1] C. Ortiz-Hernandez, A. Troncoso-Palacio, D. Acosta-Toscano, R. Begambre-Mesa y B. Troncoso-Mendoza, «Utilización de Herramientas de Calidad para la Mejora en los Procesos de Extrusión de Plásticos,» Boletín De Innovación, Logística Y Operaciones, vol. 1, nº 1, pp. 1-7, 2019.

[2] I. G. Poves-Calderón, «Aplicación de las herramientas del lean manufacturing en una empresa plástica peruana,» Universidad Peruana de Ciencias Aplicadas (UPC), 2020.

[3] C. A. Arroyo Huayta y E. S. Cruces Raimundis, «Modelo para mejorar la eficiencia en el área de extrusión de una pyme manufacturera del sector plástico basado en SMED, Mantenimiento Autónomo y 5s,» Universidad Peruana de Ciencias Aplicadas (UPC), 2020.

[4] H. M. Gonzales Pariona y B. Rocha Cam, «Modelo Lean Manufacturing de la gestión de la producción bajo un enfoque de gestión del cambio para la mejora de la productividad en una empresa dedicada a la manufactura,» Repositorio Academico UPC, 2020.

[5] A. A. Ochoa, «www.scielo.org,» 29 marzo 2007. [En línea]. Available: http://www.scielo.org.mx/scielo.php?pid=S0185-12762007000100003\&script=sci_arttext. [Último acceso: 30 Abril 2020].

[6] P. Hernández, M. Carro, J. Montes de Oca y S. Fernández, «Optimización del mantenimiento preventivo utilizando las técnicas de diagnóstico integral. Resultados parciales teórico-prácticos,» Ingeniería Energética, vol. XXIX, nº 2, pp. 26-24, 2008.

[7] H. Mao Lin, H. Tze-Haw y Z. Jiawuan, «TreemapBar: Visualizing Additional Dimensions of Data in Bar Chart,» de 13th International Conference Information Visualisation, 2009.

[8] M. L. M. Martinez Hinojosa, «www.dspace.espol.edu.ec,» 5 Enero 2008. [En línea]. Available: https://www.dspace.espol.edu.ec/handle/123456789/4510. [Último acceso: 30 Abril 2020].

[9] H. M. Ariza-sarmiento, L. M. Quevedo-Puerta y D. C. Rubio-Urueña, «Nivel de lectura de gráficos estadísticos continuos en estudiantes de la Universidad Cooperativa de Colombia,» Repositorio Institucional Universidad Cooperatiova de Colombia, 2020.

[10] A. Sánchez Comas, A. Troncoso Palacio, S. Troncoso Mendoza, and D. Neira Rodado, "Application of taguchi experimental design for identication of factors influence over 3D printing time with fused deposition modeling,'IJMSOR, vol. 1, no. 1, pp. 43-48, 2016 